\title{
MICROSTRUCTURE AND MICROTEXTURE EVOLUTION DURING HIGH TEMPERATURE LOW CYCLE FATIGUE OF NICKEL
}

\author{
S. BRODESSER \& G. GOTTSTEIN \\ Institut für Metallkunde und Metallphysik, RWTH Aachen, W-5100 Aachen, FRG
}

\begin{abstract}
The development of microstructure, texture, misorientation and grain boundary character during cyclic deformation of Nickel at elevated temperatures was investigated. At high numbers of cycles grain boundaries align with the maximum shear stress. Texture and microstructure change significantly and low $\Sigma$ CSL boundaries with $\langle 111\rangle$ rotation axes emerge. The Schmid factor increases with progressing deformation but Sachs- and Taylor-type deformation modelling fail to predict the actual texture change. It is proposed to attribute the observed texture and microstructure development to an interaction of deformation mechanisms with grain boundary motion.
\end{abstract}

KEY WORDS EBSP, high temperature low cycle fatigue, deformation modelling, grain boundary migration, grain boundary character, misorientation distribution, microtexture, Rodrigues vector.

\section{INTRODUCTION}

It has long been known that grain boundaries are liable to migration when a polycrystalline material is subjected to cyclic deformation at elevated temperatures (Yavari, P., Langdon, T. G., 1983). The phenomenon can easily be revealed by metallography, since each cycle of deformation is associated with a new position of the boundary. This is visible under the microscope due to grain boundary sliding perpendicular to the surface, which occurs concurrently with migration (Figure 1). The migration is not distributed equally among all grain boundaries, rather some boundaries move fast while other boundaries apparently do not move at all. This situation, however, may change during prolonged cyclic deformation. The migration of the boundaries does not occur at random, rather the boundaries move such as to align under $45^{\circ}$ with respect to the stress axis (Figure 2). Such boundary orientation maximizes the shear stress on the boundaries and therefore, grain boundary sliding. This in particular in two-phase materials, promotes cavity formation and, therefore, premature failure of the material under cyclic loading (Figure 3).

The situation is more complex in materials with low stacking fault energy, where dynamic recrystallization is likely to occur at high cumulative strains even for very small strain amplitudes (as low as $0.2 \%$ ). A variety of mechanisms for nucleation of dynamic recrystallization during high temperature low cycle fatigue have been demonstrated (Figure 4). In effect, dynamic recrystallization complicates the situation by introducing new grains and thus new grain boundaries into the system of grain boundaries, that undergo migration and alignment with regard to the stress axis. 


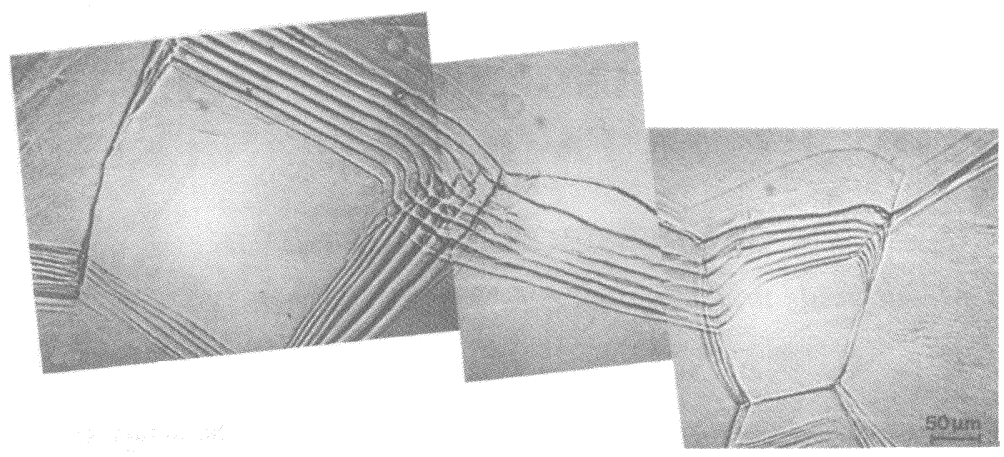

Figure 1 Grain boundary migration in Al due to cyclic deformation (Yavari, P., Langdon, T. G., 1983).

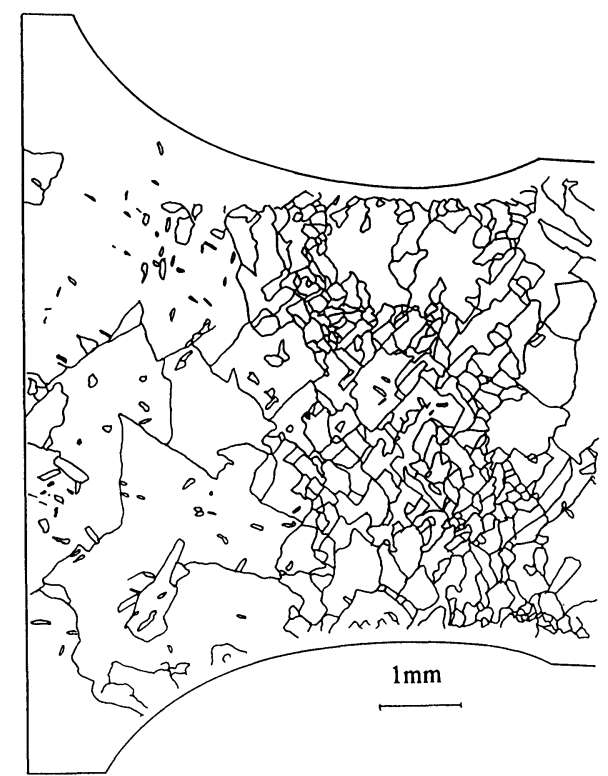

Figure 2 Microstructure of cyclically deformed $\mathrm{Ni}$ in a plane parallel to the deformation direction. 


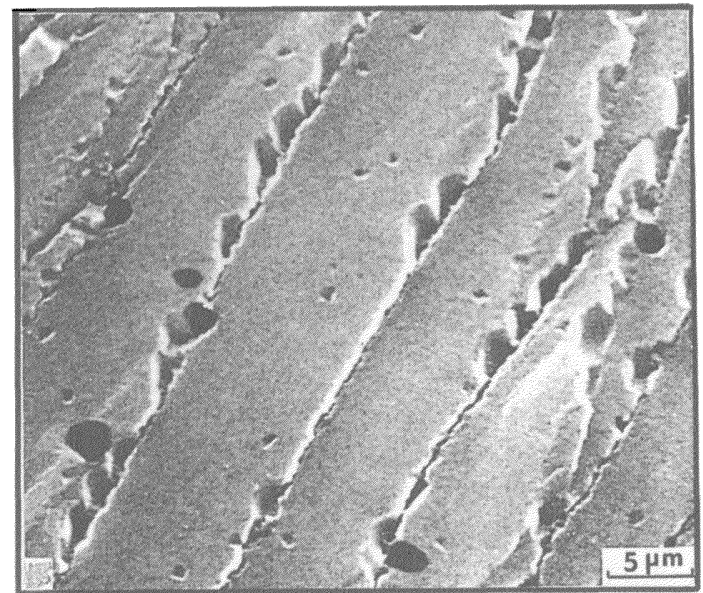

Figure 3 Cavities on grain boundaries after cyclic deformation (in Al-3\% Mg) (Raman, V., Langdon, T. G., 1989).

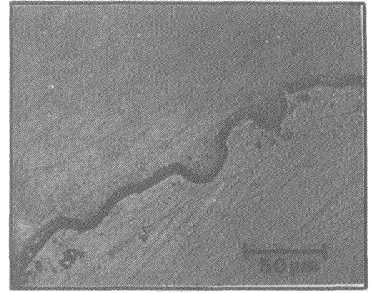

(a)

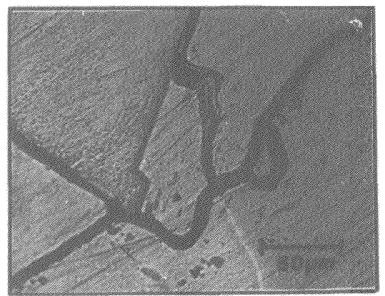

(d)

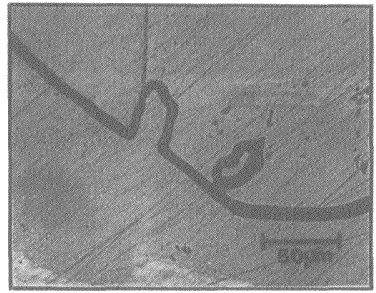

(b)

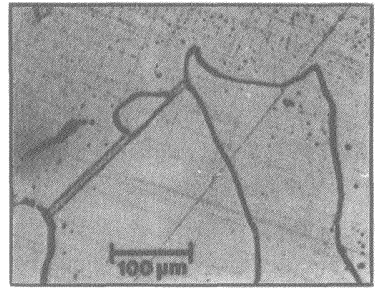

(•)

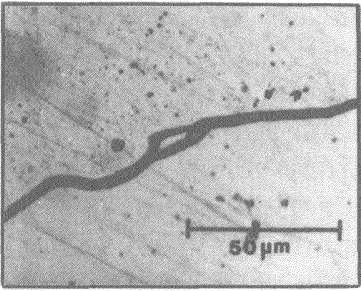

(c)

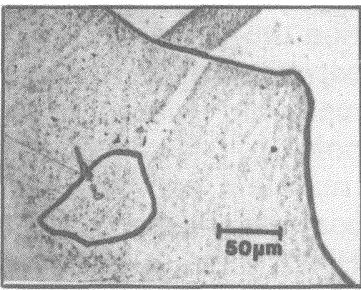

(f)

Figure 4 Mechanisms of dynamic recrystallization (Chen, S., Gottstein, G., 1988). a) Serrations of a grain boundary; b) Protrusion and new grain at a grain boundary. New grain formed c) on a grain boundary; d) near a twin; e) at a twin boundary; f) in the interior of a grain at the top of a twin. 
The phenomenon was first demonstrated on high purity lead already 30 years ago (Snowden, K. U., 1960). Although its occurrence is very obvious under a variety of experimental conditions, for instance by formation of a bamboo structure during high temperature cyclic torsion, there is a striking lack of understanding of the mechanisms that cause the migration and reorientation of the grain boundaries. It is the aim of this paper to contribute to a deeper understanding of this phenomenon by an investigation into the change of microstructure, microtexture, and misorientation between adjacent grains with progressing high temperature fatigue.

\section{EXPERIMENTAL}

From rods of pure $\mathrm{Ni}(99.95 \%)$ cylindrical specimens were machined for tension/compression low cycle fatigue tests. The specimens had a gauge section of $10 \mathrm{~mm}$ length and $4.5 \mathrm{~mm}$ diameter. Prior to deformation the samples were annealed for $5 \mathrm{~h}$ at $900^{\circ} \mathrm{C}$ in high vacuum (less than $10^{-3} \mathrm{~Pa}$ ) in order to establish a microstructure which was stable against normal grain growth or recrystallization and grain growth during deformation could be ruled out. Deformation was performed by cyclic tension/compression in high vacuum with a total (elastic and plastic) strain amplitude of $0.5 \%$ at a cyclic frequency of $13 \cdot 10^{-3} \mathrm{~Hz}$. Four equally treated specimens were deformed to $0,25,100$, and 300 cycles at $600^{\circ} \mathrm{C}$, respectively. After cooling down to room temperature (no quenching), the samples were subjected to optical metallography and microtexture evaluation. Between each processing step the macroscopic texture was measured with an automatic X-ray texture goniometer and the orientation distribution function (ODF) was computed. The orientation of individual grains was determined by means of electron back scattering patterns (EBSP) in a scanning electron microscope (SEM). All measurements were conducted on a longitudinal section through the center of the cylindric specimen. The EBSP were evaluated semi-automatically by identification of four to seven zone axes in each pattern from which the orientation of the sample was calculated according to known algorithms (Engler, O., Gottstein, G., 1992). For each sample the orientations of more than 200 grains were determined, and the misorientation between contiguous grains (between 350 and 550) were calculated. The average grain size as measured by the linear intercept method, was in the order of $150 \mu \mathrm{m}$. The measured 200 grains, therefore, represent about $20 \%$ of all grains in the cross section, i.e. a representative part of the sample. For the macrotexture determination the $\{111\},\{200\},\{220\}$ and $\{311\}$ pole figures were measured, and from the corrected intensities the ODF was calculated by means of series expansion methods. Prior to texture and microtexture measurements the samples were first polished mechanically and subsequently subjected to electropolishing. This treatment provided satisfactory imaging conditions in the SEM and good EBSP contrast. For optical microscopy the samples were etched in a solution of $40 \mathrm{ml}$ $\mathrm{HNO}_{3}, 50 \mathrm{ml} \mathrm{CH} \mathrm{COOH}_{3} \mathrm{COnd} 10 \mathrm{ml} \mathrm{H}_{2} \mathrm{O}$.

\section{RESULTS}

The change of microstructure during cyclic deformation is obvious from Figure 5. The figure is enhanced by using four different shades to distinguish four different 


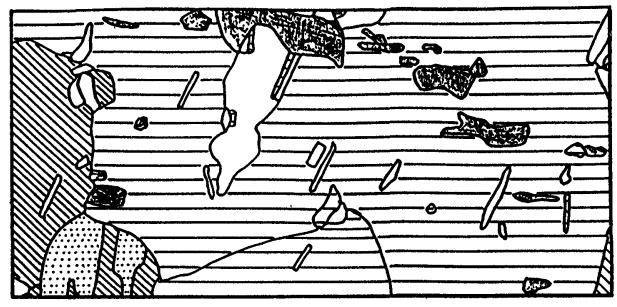

o Cycles

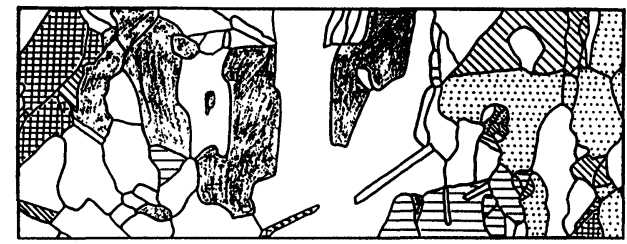

25 Cycles

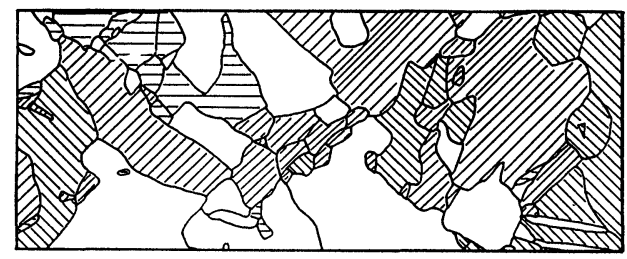

100 Cycles

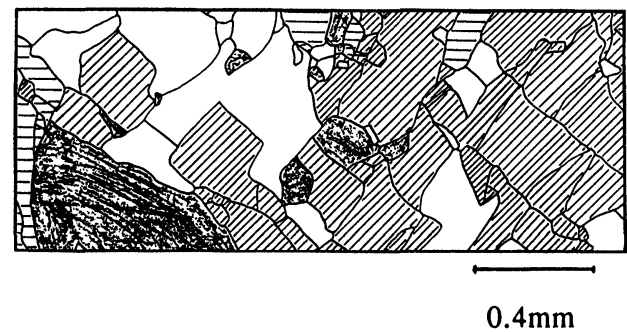

300 Cycles

Figure 5 Microstructure evolution of the specimens with progressing deformation cycles. Equally shaded areas belong to the same texture component.

texture components. Grains that belong to the same texture component are hatched identically. Comparing the microstructures with progressing stages of deformation, microstructural changes are apparent. The initial microstructure consists of very large grains with many small twins inside. It is very inhomogeneous such that there is a very large difference between minimum and maximum grain size (Figure 6). The average grain size of the starting material is $145 \mu \mathrm{m}$. After 25 cycles the average grain diameter has decreased to $130 \mu \mathrm{m}$, and the grain size has become much more homogeneous. This development is probably due to the brake-up of large grains into smaller subgrains. With increasing number of cycles the grain size increases from $145 \mu \mathrm{m}$ after 100 cycles to about $160 \mu \mathrm{m}$ after 300 cycles. Except for the initial microstructure the grains 

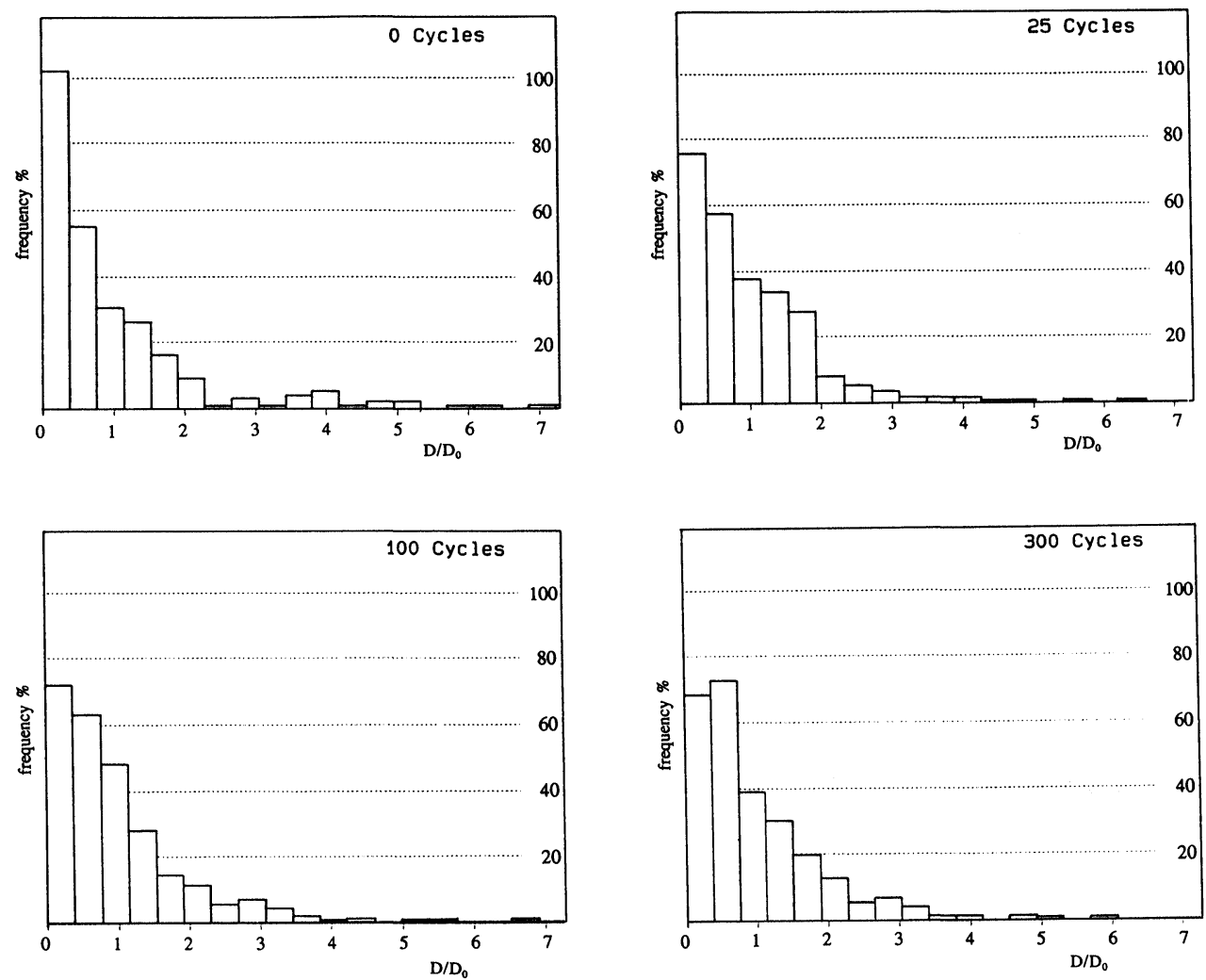

Figure 6 Change of grain size distribution with deformation.

with the maximum grain size are about 10 times larger than the smallest grains. Figure 5 confirms that the grain boundaries tend to align under $45^{\circ}$ with regard to the stress axis.

The corresponding macrotextures are represented in Euler space in Figures 7 and 8. The ODFs in Figure 7 were calculated from the measured individual grain orientations by associating with each orientation a Gauss-type orientation scatter of $10^{\circ}$ about the exact grain orientation. The grain size remained unconsidered. For the undeformed sample, the macrotexture generated this way, is composed of a strong component at $\left(88^{\circ}, 35^{\circ}, 42^{\circ}\right)$ and its twin of first order, marked as $T_{1}$ in Figure 7 , and a weak incomplete $\langle 111\rangle$ fibre texture. The macrotexture changes distinctly with progressing deformation such that first the fibre component grows at the expense of the major component at $\left(88^{\circ}, 35^{\circ}, 42^{\circ}\right)$, but later this fibre component disappears completely and a new orientation close to the cube orientation $\left(5^{\circ}, 0^{\circ}, 0^{\circ}\right)$ is formed.

The ODFs calculated from individual grain orientations compare well with the ODFs by X-ray pole figure measurements (Figure 8 ). There are, however, some noticeable quantitative differences between ODFs in Figures 7 and 8, both with regard to the height of the maxima and the exact position of the orientations. This is likely to be caused by the small number of grains measured and the grain 

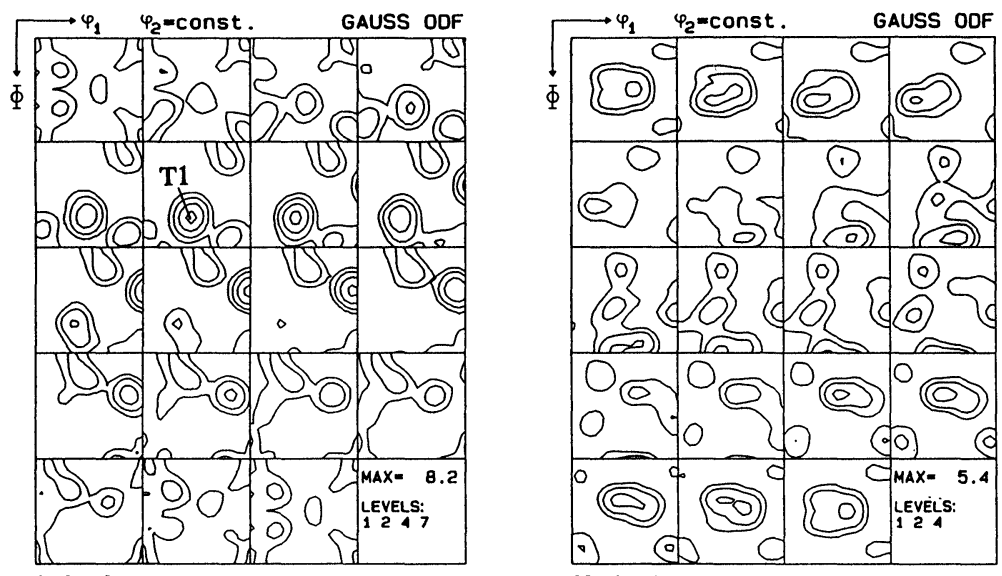

25 Cycles
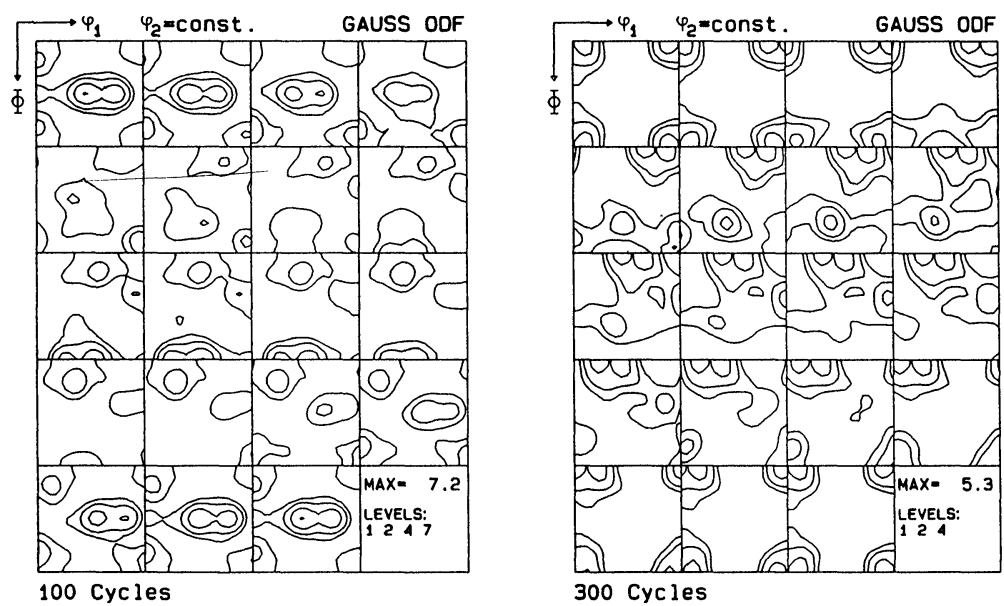

Figure 7 Gauss-ODFs of the microtextures, calculated from the single orientations with a scatter width of $10^{\circ}$.

size not being taken into account for the ODFs computed from single grain orientation measurements.

From the individual grain orientations the orientation relationship between next neighbour grains was calculated. The misorientation distribution function (MODF) is represented in Rodrigues space (Frank, F. C., 1988; Neumann, P., 1991) in Figure 9. The Rodrigues space has 3 orthogonal axes parallel to the axes of the crystal reference system. A grain misorientation is represented as a vector $\mathbf{R}$ (the Rodrigues vector) defined as

$$
\mathbf{R}=\mathbf{n} \cdot \tan \frac{\omega}{2}
$$

with $\mathbf{n}$-unit vector of rotation axis, and $\omega$-misorientation angle. For calculation 

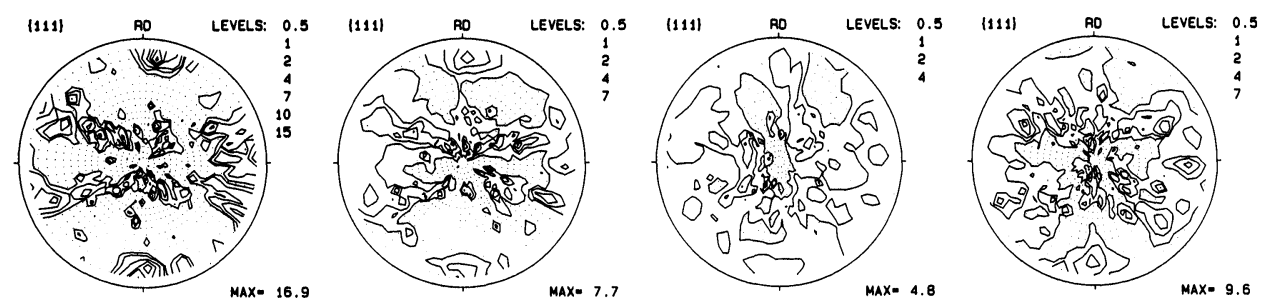

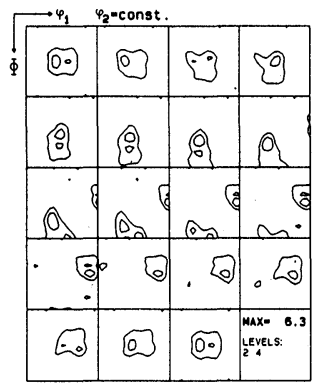

0 Cycles

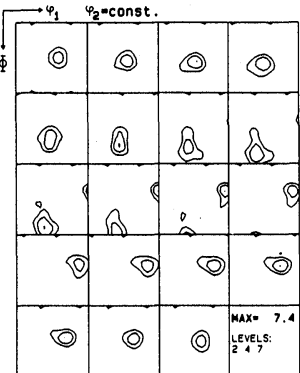

25 Cycles

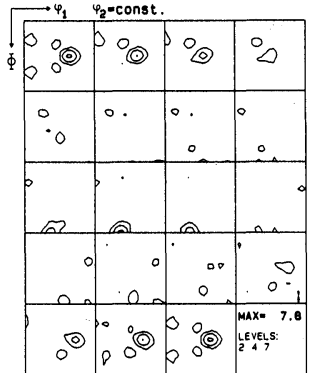

100 Cycles

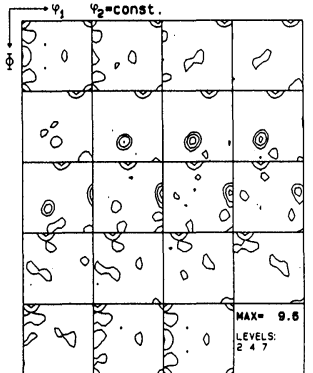

300 Cycles

Figure 8 Experimental $\{111\}$ pole figures and ODFs of the various specimens.

of the MODF each orientation was associated with a Gauss-type scatter of $2^{\circ}$ about the exact orientation relationship. For deformation up to 100 cycles the MODFs look very similar, but there are also characteristic changes. In the undeformed specimen conspicuously high orientation densities were only found close to the origin, corresponding to a frequent occurrence of small angle boundaries, first order twin orientation relationships $(\Sigma 3)$, and also second order twins $(\Sigma 9)$. The frequency of small angle boundaries attains a maximum for 25 cycles of deformation, most likely due to the evolution of a subgrain structure during deformation. Another remarkable fact is the decreasing density of first and second order twin boundaries with increasing number of deformation cycles. After 300 cycles new orientation relationships emerge, which can be associated with low $\Sigma$ coincidence site lattices (CSL), especially $\Sigma 7$ and $\Sigma 13$. In total the low $\Sigma$ boundaries $(5 \leq \Sigma \leq 25)$ comprise 13-18\% of all boundaries, including $\Sigma 1$ and $\Sigma 3$ they even amount to $50-80 \%$ of all grain boundaries.

To investigate the coupling of grain boundary orientation with applied mechanical stress, i.e. the tendency of boundaries to align parallel to the direction of maximum shear stress, the Schmid factor $\mu$ for each individual grain was determined (Figure 10). The undeformed sample shows maxima of the Schmid factor close to $\mu=0.35$ and $\mu=0.46$. The local maximum at $\mu=0.35$ is obviously due to the $\{111\}$ fibre texture of the initial material. With increasing number of cycles this local maximum diminishes, and the absolute maximum strongly increases while shifting to larger $\mu$-values. After 300 cycles a strong maximum at $\mu=0.47$ is attained.

\section{DISCUSSION}

\subsection{Microstructure}

It is evident from previous investigations and this study that the microstructure undergoes substantial changes during high temperature cyclic deformation. Grain 

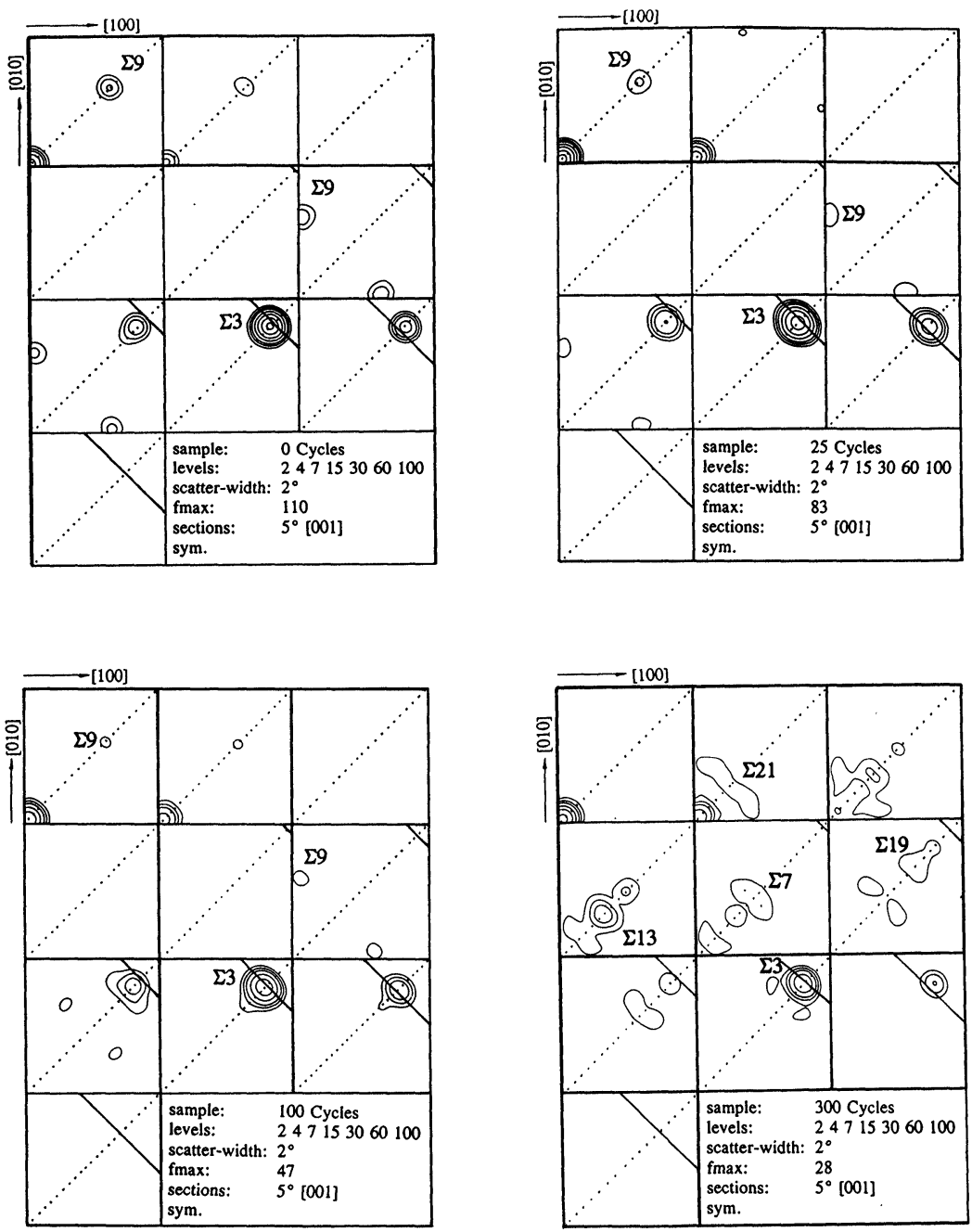

Figure 9 Misorientation distribution in Rodrigues space.

boundaries move during deformation so that an increase of the average grain size ought to be expected. The development of the grain size with increasing number of cycles, however, does not conform with this simple consideration, rather the average grain size decreases during the initial stage of deformation and noticeable grain growth is observed only at large numbers of cycles. However, the peculiarity of the initial microstructure and the evolution of the deformation substructure only pretent this development. The initial microstructure is a bimodal grain size distribution comprising few very large grains and many very small twins inside the grains (Figure 6). Neglecting the twins leads to a larger average grain size, but mixing the two vastly different sized grains would yield an average grain size not being representative for the corresponding microstructure. 

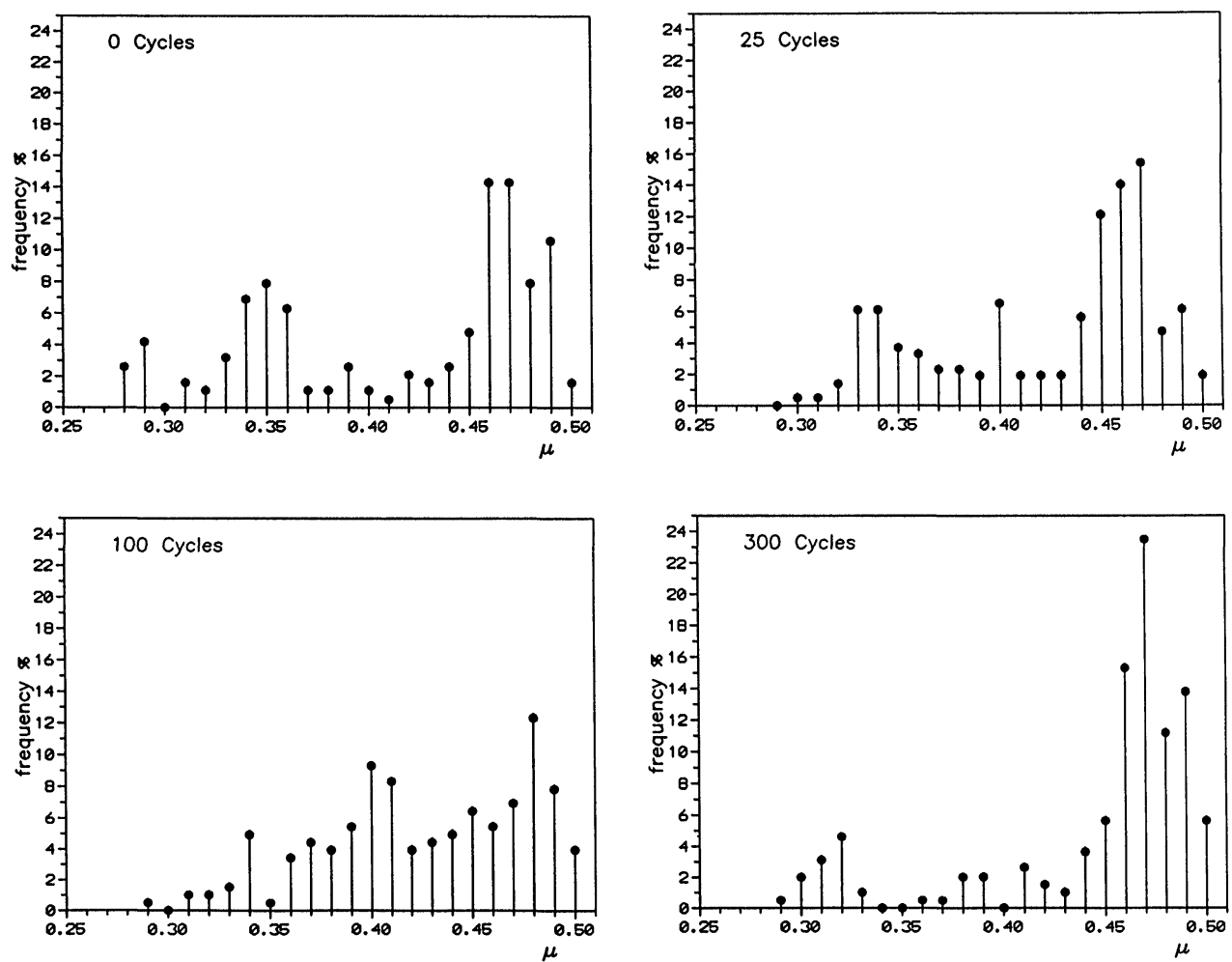

Figure 10 Distribution of Schmid factor with progressing deformation.

The decrease of grain size with increasing number of cycles during the beginning of deformation has to be attributed to a break-up of the large grains into smaller subgrains due to the evolution of a dislocation microstructure. This is evident also from the MODF (Figure 9) which shows a strong increase of small angle boundaries during the initial cycles. These small angle boundaries are the cell- or sub-boundaries of the deformation structure. It is virtually impossible, however, to distinguish these boundaries after polishing and etching under the microscope from the large angle grain boundaries. Therefore, the corresponding subgrains are treated as separate entities in the MODF.

\subsection{Texture}

The crystallographic texture measured by X-ray pole figures or by computation from single grain orientations evidently changes during deformation. Roughly the texture change can be described as a transition from an incomplete $\langle 111\rangle$ fibre texture to an incomplete $\langle 100\rangle$ fibre texture after a large number of cycles. This is obvious from the inverse pole figure of the deformed samples (Figure 11a). The inverse pole figure is a sufficient texture representation for samples with cylindrical symmetry as in the current case of uniaxial tension and compression. 
The consequence of this texture change is a noticeable increase of the average Schmid factor with increasing number of cycles. On the other hand, the change of the Schmid factor may be the driving force for the observed texture change. There are two possible approaches in terms of deformation behavior to explain the orientation change during cyclic deformation, namely by a Sachs-type or a Taylor-type deformation model. The Sachs-model assumes that the individual grains deform essentially independently, which may actually apply for the microstructure of the undeformed specimens, because the grains were very large and thus, the incompatibility of deformation at the grain boundaries was confined to a very small volume compared to the grain interior. If one assumes that the individual grains would change orientation like a single crystal under tension or compression, then the stress axis ought to rotate towards $\langle 941\rangle$ (Figure 11b). The experimental results (Figure 11a) however, do not confirm this stipulation. Although the stress axis definitely moves away from the $\langle 111\rangle$ direction, there is no accumulation of orientations close to $\langle 941\rangle$. Instead, a build-up of orientations close to $\langle 100\rangle$ can be observed, indicating the evolution of a $\langle 100\rangle$ fibre texture.

In the Taylor-model all individual grains are assumed to undergo the same, namely the macroscopic shape change. A Taylor-type simulation of up to 300
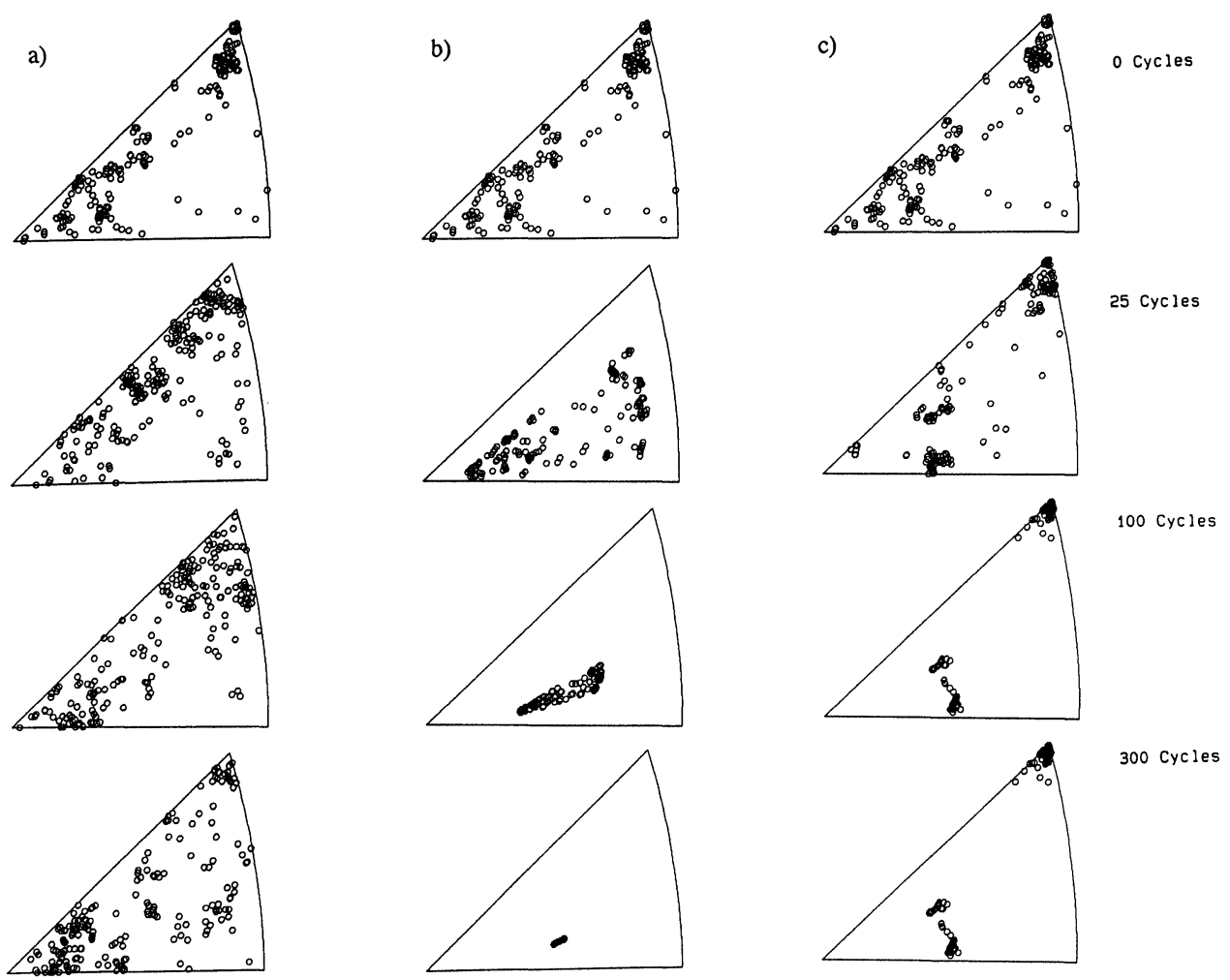

300 Cycles

Figure 11 Inverse pole figures of the stress axes of the single grain orientations a) as measured; b) calculated according to Sachs; c) calculated according to Taylor. 
cycles with $\pm 0.5 \%$ strain yields the orientation distribution given by the inverse pole figure in Figure 11c. The results predict an accumulation of orientations close to the $\langle 121\rangle$ zone like in the Sachs-model but also a stability of orientations close to $\langle 111\rangle$. Again, this is in contradiction to the experimental results. Apparently, neither a Sachs-type nor a Taylor-type deformation model conforms with the observed texture development.

There may be a quite different reason for the observed texture change, namely due to dynamic recrystallization. Dynamic recrystallization in $\mathrm{Ni}$ has been observed to be associated with annealing twin formation. $\dagger$ Annealing twins have a $60^{\circ}\langle 111\rangle$ orientation relationship to their origin. Upon twinning orientations with tension/compression axis close to $\langle 111\rangle$ relocate to the vicinity of the $\langle 100\rangle$ corner of the stereographic triangle or remain unchanged in the inverse pole figures (Figure 12). This would qualitatively conform with the observed reorientation of grains during deformation. Such an interpretation is further supported by the observation that at larger number of cycles the large grains were often found to be surrounded by relatively small grains, which are likely to be due to dynamic recrystallization. In fact, these smaller grains quite frequently reveal an orientation relationship of $60^{\circ}\langle 111\rangle$ with regard to the large grains which they

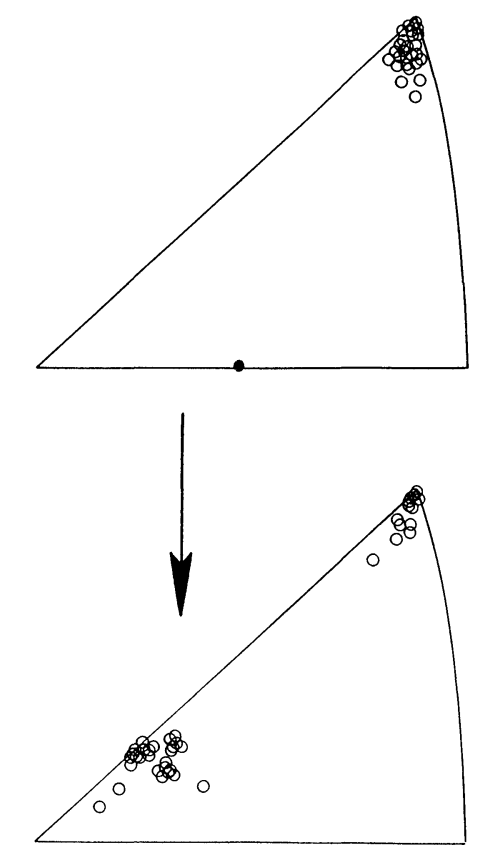

Figure 12 Change of orientation due to twinning.

$\dagger$ We like to note that twinning can also occur by grain boundary dissociation during grain boundary migration, as has been reported for grain growth in $\mathrm{Ni}$ (Lim, L. C., Ray, R., 1984). For simplicity, we subsume in this context the process of grain boundary dissociation under the phenomena of dynamic recrystallization. 
surround. This hypothesis seems to be contradictory to the finding that the density of primary and secondary twins decreases with increasing number of cycles. However, the reduction of the twin population with progressing deformation substantiates that the grains rotate during deformation such that the exact twin relationship is lost. This applies also to twins generated during deformation, which consequently do not contribute to a strengthening of the $60^{\circ}\langle 111\rangle$ misorientation components.

\subsection{Grain boundaries}

It has been proposed previously that the character of grain boundaries may be important for their ability to move during cyclic deformation. Raman, V., Watanabe, T. and Langdon, T. G. (1989) claimed from an investigation in Al that low $\Sigma$ coincidence boundaries are less liable to migration during cyclic deformation than random boundaries. However, they also found exceptions to the rule. More importantly, their investigation was limited to a very small number of cycles, and it is a frequently reported observation that some grain boundaries do not move for many cycles, but eventually become mobile. The current investigation seems to substantiate that the specimens comprise many low $\Sigma$
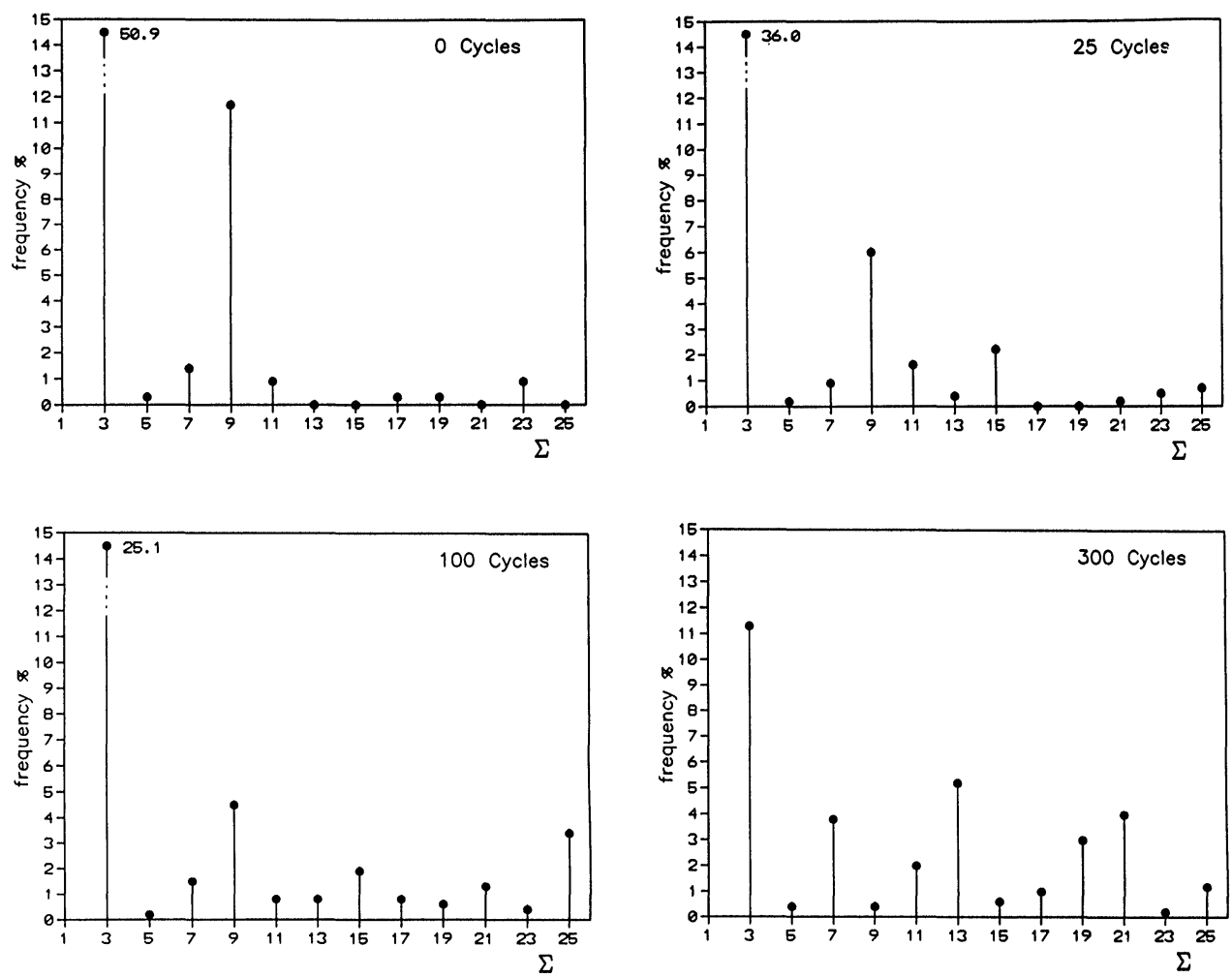

Figure 13 Frequency of $\Sigma \leq 25$ boundaries with increasing number of cycles. 
boundaries and that their distribution changes during deformation. In the undeformed sample, $80 \%$ of all grain boundaries are low $\Sigma$ boundaries with $1 \leq \Sigma \leq 25$, but $95 \%$ of all low $\Sigma$ boundaries consist of small angle boundaries $(\Sigma 1)$ and twins of first $(\Sigma 3)$ and second order $(\Sigma 9)$. Owing to the loss of twin relationships with progressing deformation the fraction of low $\Sigma$ boundaries decreases continuously to less than $50 \%$ after 300 cycles. With the decline of first and second order twin boundaries, other low $\Sigma$ boundaries become more prominent, and their fraction rises with increasing number of cycles, from $4 \%$ of the undeformed sample to $18 \%$ after 300 cycles. It is worthy to note that nearly all of these other low $\Sigma$ boundaries are characterized by $\langle 111\rangle$ rotations.

Eventually, of course, we have to address the remarkable and reproducible occurrence of grain boundary alignment under $45^{\circ}$ to the stress axes. The single grain orientation measurements yield that most of the grains with boundaries under $45^{\circ}$ with respect to the applied stress belong to the $\langle 100\rangle$ fibre component of the texture. The result is in line with the observation that the average Schmid factor is very close to 0.5 , since for a tensile axis close to $\langle 100\rangle$, the $\{111\}$ slip planes with maximum Schmid factor are oriented in a position close to $45^{\circ}$ with regard to the stress axis. Moreover, from the Rodrigues plot in Figure 9, it is apparent that most orientation relationships between neighboring grains actually correspond to $\langle 111\rangle$ rotations. If these rotation axes are perpendicular to the primary slip planes then the boundaries aligned under $45^{\circ}$ are near $\langle 111\rangle$ twist boundaries, which are known to be much less mobile than $\langle 111\rangle$ tilt boundaries. In fact, about $50 \%$ of all $45^{\circ}$ boundaries were found to be $\langle 111\rangle$ twist boundaries. These observations suggest that the alignment of the grain boundaries may be associated with both grain reorientation during deformation and orientation dependence of grain boundary mobility.

But other mechanisms cannot be ruled out. An increasing amount of grain boundary sliding may contribute to the stabilization of the boundary position parallel to maximum shear stress. When the grain boundary approaches a position parallel to the maximum shear stress, its potential for grain boundary sliding increases, while the need for dislocation glide and dislocation storage is reduced. The conspicuous increase of the Schmid factor with progressing deformation gives evidence that the deformation process does play an important role in the evolution of texture and microstructure. An increasing Schmid factor corresponds to a decreasing amount of shear for a given strain increment. Grains with low Schmid factor will suffer larger shear and therefore will store a higher dislocation density than grains with larger Schmid factor. Accordingly, there is a driving force for grain boundary migration to consume grains with small Schmid factors. This tendency diminishes with increasing contribution of grain boundary sliding to the shear strain. Sliding is favoured most, when the grain boundaries are oriented parallel to the maximum shear stress. Therefore, the observed change of texture and microstructure may also be attributed to selective grain boundary motion owing to an anisotropy of driving force. Further detailed investigations on individual grain boundaries will be necessary to clarify this important issue.

\section{CONCLUSIONS}

The microstructure and microtexture evolution during cyclic deformation of $\mathrm{Ni}$ at elevated temperatures were investigated. The microtexture was determined by 
single grain orientation determination by means of EBSP in a SEM. The following results were obtained:

(1) The microstructure was observed to undergo changes by grain boundary migration, dynamic recrystallization and grain boundary reorientation with respect to the stress axis.

(2) The microstructural changes are accompanied by changes of the orientation distribution, which converts from an incomplete $\langle 111\rangle$ to an incomplete $\langle 100\rangle$ fibre texture. A consequence of the texture change is an increase of the average Schmid factor from $\mu=0.415$ to $\mu=0.45$ after 300 cycles.

(3) The texture change cannot be explained by Sachs or Taylor type deformation models. First order twin formation during grain growth and dynamic recrystallization or selective grain growth provide a more satisfactory explanation of grain reorientation.

(4) A large fraction of the grain boundaries, ranging from $80 \%$ in the undeformed specinien to $50 \%$ after 300 cycles, could be associated to CSL boundaries with $\Sigma \leq 25$. Most of the low $\Sigma$ boundaries, however, were small angle boundaries $(\Sigma 1)$ and twin chains $(\Sigma 3, \Sigma 9)$. At higher numbers of cycles twin boundaries cease to occur, while other low $\Sigma$ boundaries emerge $(\Sigma 7, \Sigma 13, \Sigma 19, \Sigma 21)$, most of which represent $\langle 111\rangle$ rotations.

(5) The results do not yield an unambiguous interpretation of why grain boundaries align under $45^{\circ}$ to the stress axis. We surmise that the alignment of the grain boundary plane with the maximum shear stress is a combined effect of grain rotation during deformation, the anisotropy of both grain boundary mobility and driving force and a stabilization of grain boundary orientation by grain boundary sliding.

\section{References}

Chen, S. and Gottstein, G. (1988). Strain Softening, Grain Boundary Migration and Dynamic Recrystallization of Ni during High Temperature Low Cycle Fatigue, Acta metall. 36, 3093.

Engler, O. and Gottstein, G., A New Approach in Texture Research: Local Orientation Determination with EBSP, Steel Research, in press.

Frank, F. C. (1988). Orientation Mapping, ICOTOM 8 (eds. Kallend, J. S. and Gottstein, G.), TMS, Warrendale, pp. 3.

Lim, L. C. and Raj, R. (1984). On the distribution of $\Sigma$ for Grain Boundaries in Polycristalline Nickel prepared by Strain-annealing Technique, Acta metall. 32, 1177.

Neumann, P. Representation of Orientations of Symmetrical Objects by Rodrigues vectors, ICOTOM 9 , in press.

Raman, V. and Langdon, T. G. (1989). An Examination of Cyclic Grain Boundary Migration and Cavitation in an Al-3\% Mg Solid Solution Alloy, Acta metall. 37, 725.

Raman, V., Watanabe, T. and Langdon, T. G. (1989). A Determination of the Structural Dependence of Cyclic Migration in Polycrystalline Aluminium using Electron Channeling Pattern Analysis" Acta metall. 37, pp. 705.

Snowden, K. U. (1960). The Distribution of Deformation in Lead Fatigued in vacuo, Phil. Mag. 6, 321.

Yavari, P. and Langdon, T. G. (1983). An Examination of Grain Boundary Migration during High Temperature Fatigue of Aluminum-I. Microstructural Observations, Acta metall. 31, 1595. 